\title{
Biological principles of early orthodontic intervention
}

\author{
Aliakbar Bahreman* \\ Clinical professor orthodontic and pediatric dentistry programs, Eastman Institute for Oral Health, University of Rochester, NY, USA
}

For many decades, orthodontists have debated about the best age for childrent to start orthodontic treatment. Despite the American Association of Orthodontist's recommendation that orthodontic screening should be started by age 7 , many orthodontists do not treat children prior to the eruption of permanent teeth, and they postpone the treatment until the full permanent dentition at approximately age 12 .

Long-term benefits of early treatment is also controversial. In reviewing these controversies we find that the majority of debates circle around early or late treatment of class II malocclusion. We cant find as much controversy in many other services that can be performed for young patients during primary or mixed dentition, such as anterior and posterior cross bite, habit control, crowding, space management, eruption problems and many other services that a young and growing child can benefit from.

Practitioners who are in favor of early class II treatment advocate that early intervention of class II is the best choice for growth modification when the problem is skeletal and especially due to mandibular retrognathism. On the other hand, opponents believe that there is no difference in the final result, and a single-phase treatment approach is preferable because of the advantages that accompany the reduced treatment time.

The controversy surrounding early vs. late treatment is often confusing to the dental community and parents, therefore, clinicians must decide, on a case-by-case basis, when to provide orthodontic treatment. Indeed, there are occasions when delaying treatment until a later age may be advisable.

To underestand the biologic principle of early tratment requires a thorough knowledge of fundamental basics of embryology, physiology, development of dentition, and growth and development of basal bone and other skeletal structures. To answer the question "why early treatment is recommended" we have to discuse the "Mechanisms affecting occlusal development".

\section{Mechanisms affecting occlusal development}

To clarify the rationale for early-age treatment, it is necessary to describe some of the important fundamental mechanisms that can influence occlusal development such as:

$$
\begin{aligned}
& \text { * Occlusal development and craniofacial growth interaction } \\
& \text { * Genetics and environmental factors } \\
& \text { * } \text { Form and function } \\
& \text { *ocked occlusions }
\end{aligned}
$$

Occlusal development and craniofacial growth interaction: Occlusal development is a long process starting around the sixth week of intra-uterine life and concluding around the age of 20 or after.
This long developmental process is a sequence of events which occur in an orderly and timely fashion under the control of genetic and environmental factors.

Establishing a normal and harmonious arrangement of the occlusal system depends to many local and general factors including normal interaction of craniofacial growth changes and occlusal deveopmental prosess.

During long process of dentition, craniofacial growth is also interacting and we know that different part of skull grow at different rate and complete maturation differently.

Carlson [1] stated that $80 \%$ of craniofacial growth is completed by the age of 6 to 8 years, while only $50 \%$ of midfacial and mandibular growth is completed by the age of 8 to 10 years. Thus, a considerable amount of midfacial and mandibular growth potential remains during the transitional dentition that we should take advantage for growth modification.

Vertical growth of the face, as shown by Enlow [2] is the last dimension of the dento facial structure to be completed during early postnatal growth and transitional dentition. Monitoring these important growth changes and their interactions with the transitional dentition will provide us with an opportunity to detect and intervene the developing dento-skeletal deformity at proper time.

These growth potential during transitional dentition especially can help us in orthodontic and orthopedic intervention for growth modification of skeletal abnormalities such as class II class III and vertical problems.

Genetics and environmental factors: The process of occlusal development are under the influence of two basic mechanisms; genetic processes and environmental processes, which can act individually or interact with each other.

The etiology of malocclusion and the relative contribution of genes and the environment has been a matter of controversy throughout the twentieth century. Emphasizing on Genetic has been mainly based on the results of early twin and family studies. The effects of common environment from the gene-environment interaction was not separated.

Over the last 100 years, many theories have attempted to explain

Correspondence to: Aliakbar Bahreman DDS, MS, FICD, Clinical professor orthodontic and pediatric dentistry programs, Eastman Institute for Oral Health, University of Rochester, NY, USA, E-mail: Ali_Bahreman@URMC. Rochester.edu

Received: October 27, 2016; Accepted: November 07, 2016; Published: November 10, 2016 
the cause of malocclusion. Most have advocated that it is inherited, but more recently, greater emphasis has been placed on the influence of the environment, especially the activity and the posture of the oral soft tissues.

Christian [3] did an acceptable segregation of genetic from environmental effects in 1979. Harris and Johnson [4] on a study based on serial assessments of untreated person in 30 siblings from 4 years (full deciduous dentition) to 20 years of age to distinguished between skeletal variables and occlusal tooth-based variables, and concluded that while the skeletal variables had high heritability, nearly all of the occlusal variability was acquired.

Kawala B, et al. [5] assessed malocclusions susceptible to orthodontic prophylaxis by using the twin-method of analysis in monozygotic and dizygotic twins. They concluded that environmental factors account for more malocclusions than previously believed. This result encourages early intervention to intercept, disrupt, and diminish the effects of malocclusions.

Nakasima A, et al. [6] attempted to separate genetic and environmental factors related to the development of so-called pseudo and true mesioclusions. They separated this two type of mesioclusions with differential diagnosis depending on whether or not a forward mandibular shift is present or not.

The craniofacial morphogenetic characteristics of each mesioclusions were determined by lateral roentgen graphic cephalograms obtained from 66 pseudo- and 48 true mesioclusions patients and 52 control subjects, and their respective parents. They found nearly all of the significant morphogenetic differences between the two groups of patients with mesioclusion were related to environmental factors.

Recent studies [5,7,8] also indicate that Genetic factors are predominantly influencing the craniofacial morphogenesis during embryonic period, while the environmental factors affect the dento facial morphology during postnatal life particularly during transitional dentition and facial growth.

Therefore many of incipient malocclusions which are under the influence of environmental factors during primary or mixed dentition are detectable early and preventable early with better and stable result. Early recognition and interception of these factors can eliminate or reduce the severity of future problems. Examples of these common factors include:
* Early primary tooth loss
* Delayed exfoliation of primary teeth
* Over retained primary teeth
* Arch size-tooth size discrepancy
* Abnormalities of tooth number (hyperdontia and
* Eruption problems (ectopia, transposition, impaction, and
* Deleterious oral habits
* Perioral muscle dysfunction
* Abnormal frenum attachment
* Craniofacial dysfunctions, including mouth breathing, hypodontia) ankylosis) abnormal swallowing patterns, and all aspects of cranial posture

Form and function: The orofacial structure is anatomically and functionally one of the most complex regions of the human body. The phrase form and function reflects a concept of interrelation between form and function that is another important mechanism which act on orofacial structure during developing occlusion and dento-skeletal structures.

Theories on bone plasticity by Wolff [9], presumed that "Form and function are intimately related." Change in stress produce change in internal bone architecture and external bone shape. Another term of this phenomenon as was suggested by Moss ML [10] as a general principle for clinicians that becomes operant is "form follows function". The effects of muscle dysfunction during the primary or mixed dentition are not self-corrected and become worse if continued in older age. Examples of this phenomenon are:

* The activity and tension of the cheeks and lips musculatures from the outside and the tongue (size, posture, or function) from the inside are important factors, which guide the occlusal development.

If the equilibrium among these chain of muscles are interrupted, disturbances of the skeletal and occlusal development will result.

* Respiration and the capsular matrix as introduced by Moss $\mathrm{ML}^{10}$ influences the naso-maxillary complex and therefore the occlusion.

* Activity of the muscles maintaining the head position, also can affect craniofacial growth pattern and occlusion.

* Deleterious oral habits and muscle dysfunction can also disturb the dento-skeletal growth and development of occlusion.

In all of these situation early detection and preparing balanced and normal environment for dentition at early age would guide the normal development of occlusion and instability of the result.

Ovsenik $M$ [11] in a study to assess irregular orofacial functions to determine their correlation with posterior crossbite, examined 243 children at the ages 3,4 , and 5 . They found atypical swallowing, thumb sucking, and mouth breathing to be statistically significantly different between the crossbite and noncrossbite groups.

He concluded that every clinical examination of children in the deciduous dentition with sucking habits should include assessment of orofacial functions, especially the swallowing pattern, which was found to be an important factor in the etiology for posterior crossbite development. Early detection and early intervention of muscle dysfunctions is not only easily accomplished at early age treatment but it is an essential part of orthodontic treatment and a vital component for the stability of the treatment results.

In the past, clinicians such as Edward Angle [12] believed that "Orthodontic treatments are very likely to relapse, if the functional disorders are still going on.

In defense of this approach Gugino [13] pointed out that "the earlier the treatment was applied, the better the face adapted to it, whereas the longer it was deferred, the more it would have to adapt to the face".

Mahony D [14] in refining long-term orthodontic stability states that finishing the case with the muscles of mastication in equilibrium is another very important objective that often not given enough consideration. 
Locked occlusions: Some occlusal interferences, such as posterior or anterior cross bites with a mandibular shift can have an adverse effect on the normal rate and direction of jaw growth unless treated during early stages of development. These types of irregularities, are called "locked occlusions" which can cause skeletal jaw deformity if not intervened early.

Early detection and intervention of occlusal interference by mechanical unlocking of occlusion will allow normal function and therefore normal development of the occlusal system.

\section{Examples of these malocclusions include the following:}

* Locked maxillary arch, occurring during the primary or mixed dentition, which can prevent normal sagittal and transverse growth and displacement of the maxillary arch (Figure 1).

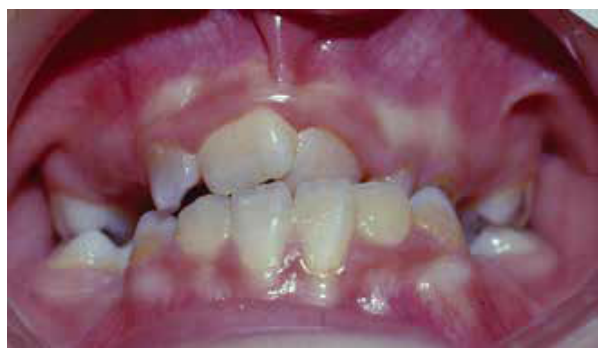

Figure 1. Locked maxillary dentition affecting normal maxillary transverse and sagittal bone growth

* Class II division one with impinging deep bite, which can cause mandibular collapse within the maxillary arch, affecting normal anterior growth of the mandible (Figure 2).

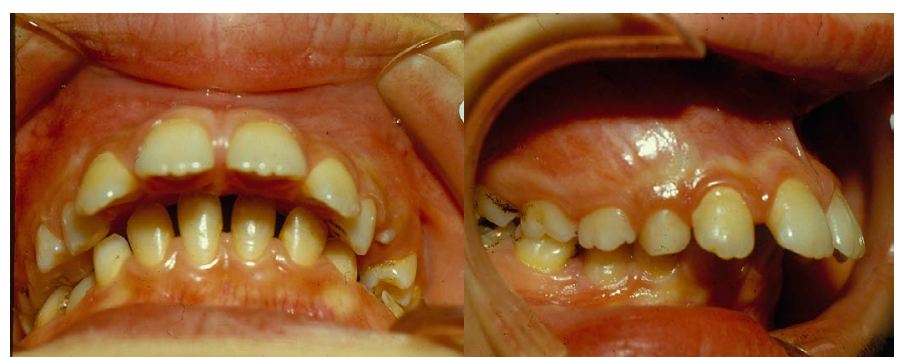

Figure 2. Locked mandibular arch within maxilla preventing normal mandibular sagitta growth.

* Unilateral cross bite with a functional shift, which is a common problem in primary and early mixed dentition and, if not treated at an early age, can affect mandibular growth, causing asymmetric jaw growth. Figure 3 shows a neglected case with posterior cross bite and mandibular shift to the left, not treated early causing asymmetric mandibular growth and facial asymmetry.

Both functional and mechanical unlocking of this type of malocclusions cannot be overlooked in orthodontic diagnosis and treatment planning. Most of investigatores for posterior crossbite with mandibulare shift recommend early intervention during primary or early mixed dentition.

\section{Conclusion}

* Understanding of biological principle of developing malocclusion and morphogenesis of abnormalities of dentition can guide us in early detection and proper interventions.

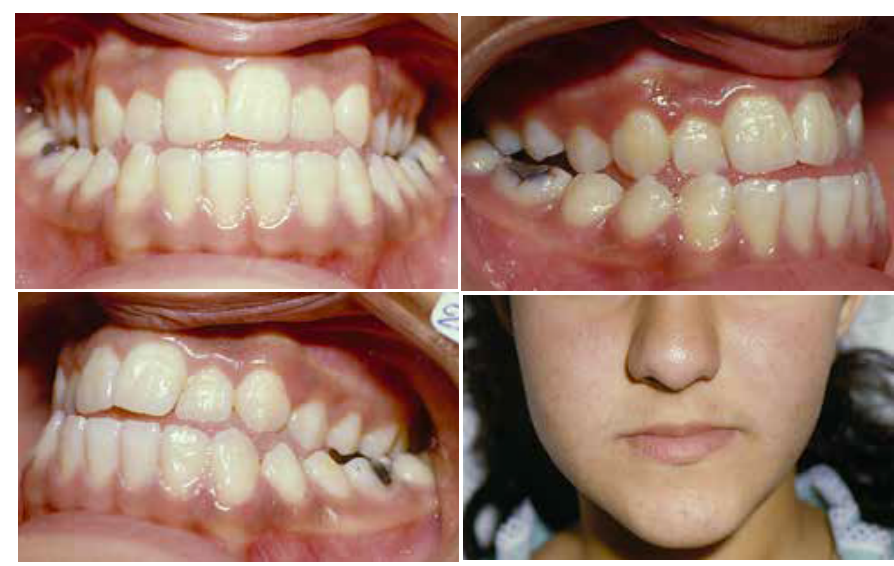

Figure 3. Posterior crossbite causing mandibular left lateral shift.

* These type of treatment are often initiated in the developing dentition to promote favorable developmental changes and remove or suppress those that are unfavorable to normal development of dentition.

* Early interceptive orthodontics can eliminate or reduce the severity of a developing malocclusion, the complexity of orthodontic treatment, and overall treatment time and cost.

\section{Disclosure}

The author is hopping by presenting this article and discussing the most important principle of occlusal development, can answer the first question "why early age orthodontic intervention is recommended". Those readers who are intrested in clinical application of the content of this article regarding the the two other common questions (1. when and 2. how early treatment can be applied) can refer to my book "Early age orthodontic treatment" [15] published by Queintessence publisher, in April 2013.

\section{References}

1. Carlson DS (2002) Biological rationale for early treatment of dentofacial deformities Am J Orthod Dentofacial Orthop 121: 554-558. [Crossref]

2. Enlow D. Handbook of facial growth, ed 2 Philadelphia: Saunders, 1982

3. Christian JC (1979) Testing twin means and estimating genetic variance: Basic methodology for the analysis of quantitative twin data. Acta Genet Med Gemellol (Roma) 28: 35-40.

4. Harris EF, Johnson MG (1991) Heritability of craniometric and occlusal variables: a longitudinal sib analysis. Am J Orthod Dentofacial Orthop 99: 258-268. [Crossref]

5. Kawala B, Antoszewska J, Necka A (2007) Genetics or environment? A twin-method study of malocclusions. World J Orthod 8: 405-410. [Crossref]

6. Nakasima A, Ichinose M, Nakata S (1986) Genetic and environmental factors in the development of so-called pseudo- and true mesiocclusions. Am J Orthod Dentofacial Orthop 90: 106-116. [Crossref]

7. Ovsenik M (2009) Incorrect orofacial functions until 5 years of age and their association with posterior crossbite. Am J Orthod Dentofacial Orthop 136: 375-381. [Crossref]

8. John R.C. Mew, BDS Lond, LDS RCS Eng, MFGDP (UK), M.Orth RCS Edin: The postural basis of malocclusion: A philosophical overview, American Journal of Orthodontics and Dentofacial Orthopedics, Volume 126, Issue 6, December 2004, Pages 729-738

9. 9. Wolff J. "The Law of Bone Remodeling". Berlin Heidelberg New York: Springer 1986 (translation of the German 1892 edition).

10. 10. Moss ML. The functional matrix. Vistas in orthodontics. Philadelphia: Lea \& Febiger; 1962

11. Ovsenik M (2009) Incorrect orofacial functions until 5 years of age and their association with posterior crossbite. Am J Orthod Dentofacial Orthop 136: 375-381. [Crossref] 
12. Edward Angle. Treatment of malocclusion of the teeth (7th Ed) S. S. White, Philadelphia 1907.

13. Gugino CF, Dus I (1998) Unlocking orthodontic malocclusions: an interplay between form and function. Semin Orthod 4: 246-255. [Crossref]
14. Mahony D (2005) Refining occlusion with muscle balance to enhance long-term orthodontic stability. J Clin Pediatr Dent 29: 93-98. [Crossref]

15. Ali A. Bahreman "Early age orthodontic treatment" Quintessence publisher April 2013.

Copyright: (C2016 Bahreman A. This is an open-access article distributed under the terms of the Creative Commons Attribution License, which permits unrestricted use, distribution, and reproduction in any medium, provided the original author and source are credited. 